\title{
An electrically injected InAs/GaAs quantum-dot photonic crystal microcavity light-emitting diode
}

\author{
J. Sabarinathan, P. Bhattacharya, ${ }^{\text {a) }}$ P-C. Yu, and S. Krishna ${ }^{\text {b) }}$ \\ Solid State Electronics Laboratory, Department of Electrical Engineering and Computer Science, \\ University of Michigan, Ann Arbor, Michigan 48109-2122 \\ J. Cheng, D. G. Steel \\ Harrison M. Randall Laboratory of Physics, University of Michigan, Ann Arbor, Michigan 48109-2122
}

(Received 25 July 2002; accepted 20 September 2002)

\begin{abstract}
An electrically injected InAs/GaAs self-organized quantum-dot photonic crystal microcavity light-emitting diode operating at $1.04 \mu \mathrm{m}$ is demonstrated. Light-current characteristics are obtained for devices with two- and five-defect period cavities with maximum light output of 0.17 $\mu \mathrm{W}$ measured in the surface-normal direction. Near-field images were also obtained for an injection current of $8.35 \mathrm{~mA}$, showing light confinement within a few periods of the photonic crystal defect microcavity. (C) 2002 American Institute of Physics. [DOI: 10.1063/1.1521249]
\end{abstract}

Since the realization that spontaneous emission can be altered by controlling the environment in which the radiating element is placed, ${ }^{1}$ efforts have been made to produce submicron cavities in order to control the spontaneous emission process in the optical regime. Quantum dot (QD) microcavity light-emitting diodes (LEDs) offer some unique advantages, including carrier and photon confinement in the same region, small optical loss, and a closer match of the narrow QD emission linewidth with the near-singular photon density of states. Such a device would also be a good vehicle to study effects related to quantum entanglement. Optically pumped photonic crystal microcavities with QD active regions have been recently demonstrated by other groups., We describe here the design, fabrication, and characterization of an electrically injected surface emitting photonic crystal defect microcavity LED with self-organized InAs/ GaAs QDs emitting at $1.04 \mu \mathrm{m}$ as the recombination medium in the microcavity.

The design of the photonic crystal (PC) defect microcavity is initiated with a suitable choice of the fill factor of the photonic crystal lattice determined by the ratio $r / a$, where $r$ is the radius of the air-holes and $a$ is the lattice constant of the triangular lattice structure. ${ }^{4}$ In our design, $r / a=0.35$ was chosen. The operating wavelength $\lambda$ is fixed by the vertical cavity design and the QD active region emission, which was at $1.04 \mu \mathrm{m}$ at $300 \mathrm{~K}$ for the InAs/GaAs QDs in the heterostructure device. With $\lambda$ and $r / a$ fixed, the next step is to determine the band structure of the photonic crystal for TE modes and the normalized frequency $a / \lambda$ of the defect mode within the band gap. The finite thickness of the twodimensional (2D) photonic crystal in the vertical direction, which for our $\lambda$-cavity structure is $d=0.39 \mu \mathrm{m}$, has to be considered to accurately calculate the photonic crystal band structure. The simulations were done using the effective index method along with plane wave expansion techniques. ${ }^{5,6}$

\footnotetext{
${ }^{a)}$ Electronic mail: pkb@eecs.umich.edu

b) Now at: Center for High Technology Materials (EECE Dept.), University of New Mexico, Rm. 118A, 1313 Goddard Street SE, Albuquerque, NM 87106.
}

The vertical cavity in our device formed by a dielectric-air interface on top and a distributed Bragg reflector (DBR) in the bottom was simulated as a slab of height $0.39 \mu \mathrm{m}$ with air on the top and a homogeneous medium with averaged index of refraction of 1.7 in the bottom, determined by the penetration depth of the guided mode in the DBR region. The effective index calculations for this $\lambda$-cavity slab thus yield two modes (corresponding to two effective indices) with the second-order mode of the structure giving an effective index $n_{\text {eff }}=1.75$. This value was subsequently used to calculate the band-structure of the photonic crystal using 2D plane wave expansion technique. As shown in Fig. 1, we have a band gap for the second-order mode for normalized frequency range $a / \lambda$ between 0.39 and 0.43 . The normalized frequency $a / \lambda=0.41$ within this band gap is chosen for our design encompassing the peak emission of the QDs at $\lambda=1.04 \mu \mathrm{m}$ and fill factor $r / a=0.35$. The final design values for the photonic crystal are $a=0.42 \mu \mathrm{m}$ and $r=0.15 \mu \mathrm{m}$.

The GaAs-based device heterostructure, shown in Fig. 2(a), was grown by molecular beam epitaxy (MBE). Crosssectional transmission electron microscopy has revealed that a single self-organized InAs/GaAs QD is approximately py-

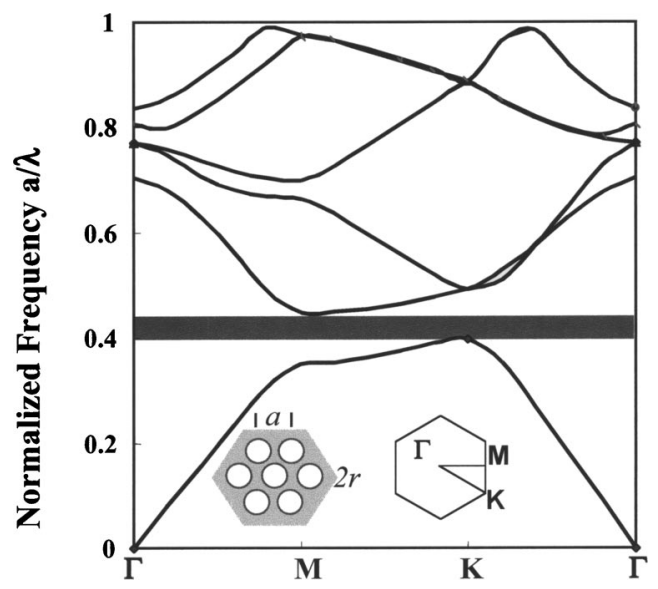

FIG. 1. Calculated band structure of photonic crystal slab using the effective index method where the effective index $n_{\text {eff }}=1.8$ for the second-order guided mode in the $\lambda$-cavity slab and plane wave expansion technique. 


\begin{tabular}{|c|c|c|c|}
\hline GaAs & $0.0746 \mu_{\mathrm{m}}$ & $\mathrm{p}+$ & $2 \times 10^{19}$ \\
\hline $\mathrm{Al}_{0.8} \mathrm{Ga} a_{0.2} \mathrm{As}$ & $0.0854 \mu_{\mathrm{m}}$ & $\mathrm{p}$ & $2 \times 10^{18}$ \\
\hline GaAs & $0.0746 \mu_{\mathrm{m}}$ & $\mathrm{p}$ & $2 \times 10^{18}$ \\
\hline $\mathrm{Al}_{0.96} \mathrm{Ga}{ }_{0.04} \mathrm{As}$ & $0.0879 \mu_{\mathrm{m}}$ & $\mathrm{p}$ & $2 \times 10^{18}$ \\
\hline GaAs & $0.1396 \mu_{\mathrm{m}}$ & $\mathrm{i}$ & ---- \\
\hline InGaAs dots & QD & $\mathbf{i}$ & --- \\
\hline GaAs barrier & 20 & $\mathrm{i}$ & --- \\
\hline InGaAs dots & QD & $\mathrm{i}$ & -.--- \\
\hline GaAs Spacer & $0.1396 \mu_{\mathrm{m}}$ & i & -- \\
\hline $\mathrm{Al}_{0.96} \mathrm{Ga}_{0.04} \mathrm{As}$ & $0.0879 \mu_{\mathrm{m}}$ & $\mathrm{n}$ & $2 \times 10^{18}$ \\
\hline GaAs & $0.0746 \mu_{m}$ & $\mathrm{n}$ & $2 \times 10^{18}$ \\
\hline $\mathrm{Al}_{0.8} \mathrm{Ga}_{0.2} \mathrm{As}$ & $0.0854 \mu_{\mathrm{m}}$ & $\mathrm{n}$ & $2 \times 10^{18}$ \\
\hline
\end{tabular}

(a)

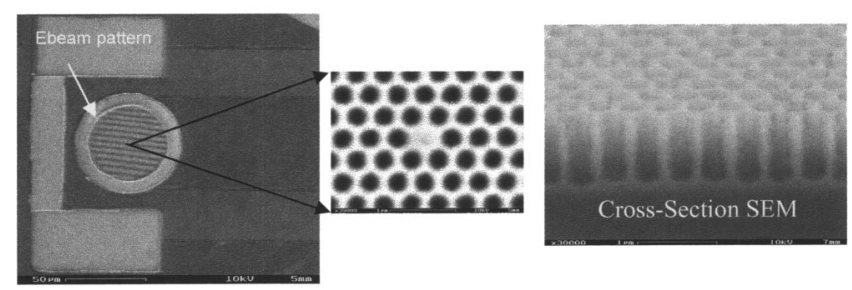

(b)

FIG. 2. (a) Device heterostructure grown by MBE on $n^{+}$GaAs substrate with five InAs/GaAs QD layers in a $\lambda$-cavity; (b) plan view SEM of surface emitting device after standard processing with single defect photonic crystal e-beam pattern integrated in the center of the aperture and cross-sectional SEM of single defect photonic crystal after e-beam patterning, pattern reversal, and deep etching with hole radius of $0.15 \mu \mathrm{m}$, a pitch of $0.42 \mu \mathrm{m}$, and a depth of $1.0 \mu \mathrm{m}$.

ramidal in shape with base length and height of $20 \mathrm{~nm}$ and 6 $\mathrm{nm}$, respectively. ${ }^{7}$ It may be noted that the resonant defect mode is highly localized around the defect and can either propagate in the plane of the photonic crystal by tunneling, or leak out in the vertical direction. The heterostructure samples were then heat treated by rapid thermal annealing at $740{ }^{\circ} \mathrm{C}$ for $40 \mathrm{~s}$. The annealing was performed to blueshift the room temperature emission peak of the as-grown QDs from $1.09 \mu \mathrm{m}$ to the design wavelength of $1.04 \mu \mathrm{m}$, improve the emission intensity and decrease the emission linewidth.

The photoluminescence (PL) of the annealed InAs/GaAs QDs, before fabrication of the photonic crystal and the rest of the device, was measured at $17 \mathrm{~K}$ using an $\mathrm{Ar}+$ laser source, a monochromator, and liquid nitrogen cooled photomultiplier tube. Transitions represented by a peak and a shoulder are observed at 1.01 and $1.025 \mu \mathrm{m}$ with a separation of $20 \mathrm{meV}$, as shown in Fig. 3. We believe that these two peaks, respectively, correspond to transitions related to the excited and ground states of the QDs. The separation between the transition peaks is smaller than that for as-grown dots, typically $\sim 60 \mathrm{meV}$, due to intermixing-related effects in the dot heterostructure caused by annealing. ${ }^{8}$ In addition, a stronger intensity of the excited state transition is characteristic of annealed quantum dots. ${ }^{8}$ It may be noted that the spectral shift of QD transitions with temperature is smaller in QDs, compared to bulk semiconductors. Therefore, we expect the $1.025 \mu \mathrm{m}$ transition to shift to $\sim 1.04 \mu \mathrm{m}$ at $300 \mathrm{~K}$. After the device fabrication and formation of the photonic crystal, microPL measurements were performed using a 5 $\mathrm{mW} \mathrm{Ar}+$ laser source at $8 \mathrm{~K}$. Figure 3 shows the measurements for a five-defect cavity sample, and we notice two peaks, at 1.015 and $1.035 \mu \mathrm{m}$, of almost equal intensity.

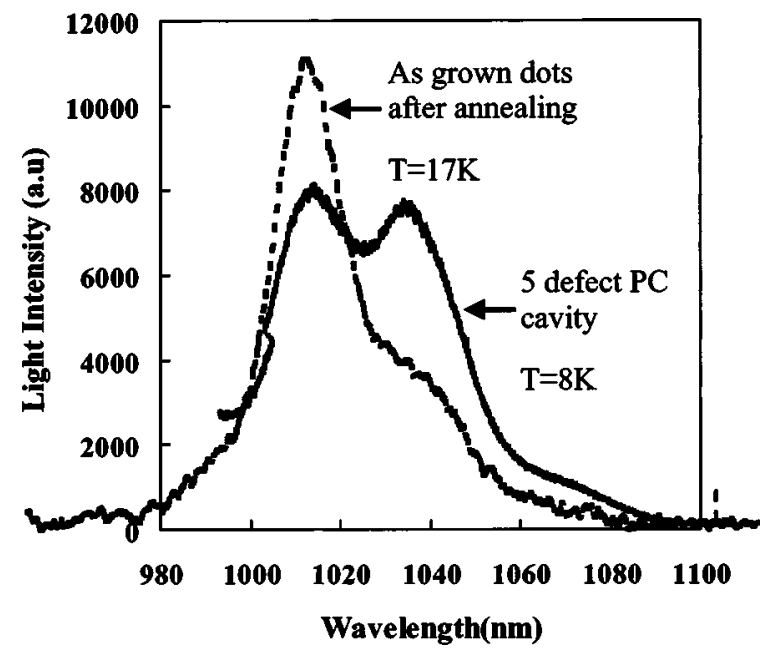

FIG. 3. PL of as-grown InAs/GaAs QDs at $17 \mathrm{~K}$ after annealing and micro-PL of QDs in a five-defect period photonic crystal microcavity at $8 \mathrm{~K}$.

Comparing with the as-grown PL. we see that the stronger excited state transition of the sample before fabrication of the PC is suppressed while the weaker ground state transition is enhanced. This is probably due to the microcavity effect of the PC and the $1.035 \mu \mathrm{m}$ transition is in resonance with one of the dominant cavity modes.

Standard etching, metallization, and photolithography steps were used in initiating the device processing. The 2D photonic crystal formation is achieved subsequently by e-beam lithography, pattern transfer, and deep dry etching $(0.8 \mu \mathrm{m})$ techniques. Figure 2(b) shows the plan view and cross-sectional scanning electron microscope (SEM) images of the devices after fabrication of the photonic crystal microcavity. Devices were fabricated having 1-10 defects forming the microcavity. The single defect microcavity has a diameter of $0.54 \mu \mathrm{m}$. From a QD density of $5 \times 10^{10} \mathrm{~cm}^{2}$, determined by atomic force microscopy on similar control samples, we estimate that there are approximately 500 dots in the microcavity.

The devices were tested in pulsed-mode $(1 \mu$ s with $1 \%$ duty cycle) with heat sinking. The light-current $(L-I)$ characteristics of two- and five-defect microcavity devices are shown in Fig. 4. The emission in the surface normal direction is measured. The output intensity is also very small. This could be due to many reasons, including the small recombination volume provided by $\sim 500$ dots, defects in and around the dots, and imperfections in device processing, giving rise to surface recombination. A maximum light output of 0.17 $\mu \mathrm{W}$ is measured from a single two-defect device, which indicates that the etching of the air holes produces reasonably high quality etch surfaces. Nonetheless, the output efficiency is extremely small, $1.4 \times 10^{-3} \%$, and there is substantial room for improvement.

It should be noted that the light measured in the vertical direction could possibly have two components. One component is the in-plane mode due to the photonic crystal, which leaks out vertically and which we have designed the cavity for. The other component of the measured light also present, arises from photons propagating vertically between the airsemiconductor interface and the bottom DBR. Cavities with 


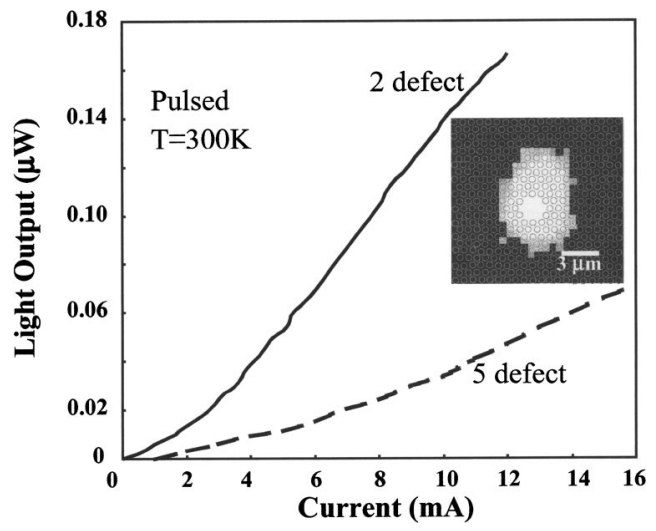

FIG. 4. Light-current characteristics in pulsed-mode $(1 \mu$ s with $1 \%$ duty cycle) of photonic crystal two- and five-defect period microcavity devices with InAs/GaAs QD active region emitting at $1.04 \mu \mathrm{m}$ at room temperature with heat sinking; inset shows the near-field image of two-defect period microcavity $1.38 \mu \mathrm{m}$ surface diameter under $7.15 \mathrm{~mA}$ injection measured at a distance of $4 \mathrm{~mm}$ from the device surface. The image extending to $6 \mu \mathrm{m}$ is shown superimposed on the photonic crystal lattice. It is evident that the light originates from and is confined to, at best, a few periods of the photonic lattice, while the oxide-defined window extends to 50 periods.

different $r / a$ (fill factor) were also fabricated to compensate for variations due to fabrication. We obtained measurable light output from devices at a particular $r / a$ value only, which indicates that the light characteristics are most probably related to the photonic crystal. We believe that the inplane mode is the dominant mode due to the $r / a$ dependence of the light output. It is also noticed in Fig. 4 that the output of the two-defect device is larger than that of the five-defect device. We have consistently recorded this trend in similar quantum well devices. At this point we can cautiously attribute it to weak microcavity effects.

The near-field image of the light output was measured with a Spiricon Laser Beam Diagnostics system for injection currents ranging from $2.85-8.35 \mathrm{~mA}$. The inset of Fig. 4 shows the output for an injection current of $7.15 \mathrm{~mA}$ injection. The imaging was done at a distance of $4 \mathrm{~mm}$ from the surface of the device through an objective lens. It is important to note that even at this distance, where some amount of spreading takes place, the extent of the near-field image is several times smaller than the 50- $\mu$ m-diameter device aperture defined by wet oxidation of $\mathrm{Al}_{0.96} \mathrm{Ga}_{0.04} \mathrm{As}$. There is also the possibility of light scattering in the air holes and diffraction at the surface. One has also to consider the possibility that we see light output from the central defect region because the patterned region is optically "darker" due to surface recombination. However, the injected holes from the top $p$-contact have to reach the active region by traversing around the etched holes. It is inconceivable that carriers traveling to the center defect region encounter fewer surface states than in traveling to the surrounding regions. The results are therefore very encouraging and indicate that electrically injected photonic crystal microcavity light sources can be realized by our processing technique.

The authors thank Dr. H. Everitt for his interest. This work is being supported by the Army Research Office under Grant DAAD 19-01-1-0527.

${ }^{1}$ E. M. Purcell, Phys. Rev. 69, 681 (1946).

${ }^{2}$ C. Reese, C. Becher, A. Imamoglu, E. Hu, B. Gerardot, and P. Petroff, Appl. Phys. Lett. 78, 2279 (2001).

${ }^{3}$ T. Yoshie, A. Scherer, H. Chen, D. Huffaker, and D. Deppe, Appl. Phys. Lett. 79, 114 (2001)

${ }^{4}$ J. D. Joannopoulos, R. D. Meade, and J. N. Winn, Photonic Crystals (Princeton, New York, 1995).

${ }^{5}$ C. Pollock, Fundamentals of Optoelectronics (Irwin, Chicago, 1995).

${ }^{6}$ R. D. Meade, A. M. Rappe, K. D. Brommer, J. D. Joannopoulos, and O. L. Alerhand, Phys. Rev. B 48, 8434 (1993).

${ }^{7}$ P. Bhattacharya, K. K. Kamath, J. Singh, D. Klotzkin, J. Phillips, H. T. Jiang, N. Chervela, T. B. Norris, T. Sosnowski, J. Laskar, and M. R. Murty, IEEE Trans. Electron Devices 46, 871 (1999).

${ }^{8}$ T. M. Hsu, Y. S. Lan, W. H. Chang, N. T. Yeh, and J. I. Chyi, Appl. Phys. Lett. 76, 691 (2000) 